\title{
Sedentary behaviour in relation to ovarian cancer risk: a systematic review and meta-analysis
}

\author{
Veronika S. Biller ${ }^{1}$ (D . Michael F. Leitzmann ${ }^{1} \cdot$ Anja M. Sedlmeier $^{1} \cdot$ Felix F. Berger $^{1} \cdot$ Olaf Ortmann $^{2} \cdot$ Carmen Jochem $^{1}$
}

Received: 4 September 2020 / Accepted: 15 December 2020 / Published online: 25 January 2021

(c) The Author(s) 2021

\begin{abstract}
Sedentary behaviour is an emerging risk factor for several site-specific cancers. Ovarian cancers are often detected at late disease stages and the role of sedentary behaviour as a modifiable risk factor potentially contributing to ovarian cancer risk has not been extensively examined. We systematically searched relevant databases from inception to February 2020 for eligible publications dealing with sedentary behaviour in relation to ovarian cancer risk. We conducted a systematic review and meta-analysis, calculating summary relative risks (RR) and 95\% confidence intervals (CI) using a random-effects model. We calculated the E-Value, a sensitivity analysis for unmeasured confounding. We tested for publication bias and heterogeneity. Seven studies (three prospective cohort studies and four case-control studies) including 2060 ovarian cancer cases were analysed. Comparing highest versus lowest levels of sedentary behaviour, the data indicated a statistically significant increase in the risk of ovarian cancer in relation to prolonged sitting time $(\mathrm{RR}=1.29,95 \% \mathrm{CI}=1.07-1.57)$. Sub-analyses of prospective cohort studies ( $\mathrm{RR}=1.33,95 \% \mathrm{CI}=0.92-1.93)$ and case-control studies $(\mathrm{RR}=1.28,95 \% \mathrm{CI}=0.98-1.68)$ showed statistically non-significant results. Sensitivity analysis showed that an unmeasured confounder would need to be related to sedentary behaviour and ovarian cancer with a RR of 1.90 to fully explain away the observed RR of 1.29. Our analyses showed a statistically significant positive association between sedentary behaviour and ovarian cancer risk.
\end{abstract}

Keywords Sedentary behaviour $\cdot$ Ovarian cancer $\cdot$ Meta-analysis $\cdot$ Systematic review

\section{Introduction}

Ovarian cancer is the eighth most common cancer in women worldwide, with 295,414 incident cases and 184,799 deaths in 2018 [1]. Due to non-specific symptoms and a lack of effective screening methods, ovarian cancer is often detected at an advanced stage, which results in poor survival rates $[2,3]$.

Sedentary behaviour is a modifiable risk factor for several chronic diseases including different tumour entities [4]. Sedentary behaviour is defined as "any waking behaviour

Supplementary Information The online version of this article (https://doi.org/10.1007/s10654-020-00712-6).

Veronika S. Biller

veronika.biller@stud.uni-regensburg.de

1 Department of Epidemiology and Preventive Medicine, University of Regensburg, Regensburg, Germany

2 Department of Gynaecology and Obstetrics, University of Regensburg, Regensburg, Germany characterized by an energy expenditure $\leq 1.5$ metabolic equivalents (METs), while in a sitting, reclining or lying posture" [5]. Individuals spend more than half of their daily waking hours sedentary, e.g. while watching television (TV), using means of transport, at work or in school [6]. Importantly, sedentary behaviour is distinct from physical inactivity since someone can meet the physical activity recommendations despite being highly sedentary throughout the remaining waking hours [7].

Several epidemiologic studies have investigated the association between sedentary behaviour and ovarian cancer risk. Previous meta-analyses generated inconsistent findings [4, 8-10]. Whereas two initial meta-analyses published in 2014 yielded positive but statistically non-significant results [4, 10], two recent meta-analyses showed a statistically significant positive association between prolonged sitting time and ovarian cancer risk $[8,9]$. It is noteworthy that none of the meta-analyses included all available studies with ovarian cancer as the primary outcome and the majority assessed multiple cancer types $[4,9,10]$. The most recent meta-analysis did not exclusively investigate sedentary behaviour as 
exposure and entailed several methodological limitations [8]. Therefore, an up-to-date and comprehensive systematic review and meta-analysis focusing on sedentary behaviour in relation to ovarian cancer risk is needed.

\section{Methods}

The present study was performed according to the Preferred Reporting Items for Systematic Reviews and Meta-Analyses (PRISMA) [11]. The PRISMA checklist can be accessed online (Online Resource 1).

\section{Inclusion criteria}

To qualify for our analysis, studies had to fulfil the following inclusion criteria: studies 1) represented a cohort or case-control design, 2) were conducted in adult women, 3) defined total sitting time, leisure-time sitting, TV-viewing time or occupational sitting time as an exposure variable and considered ovarian cancer risk as the primary outcome, 4) provided a relative risk (RR), odds ratio (OR) or hazard ratio (HR) and 95\% confidence intervals (CI) for highest versus lowest levels of sedentary behaviour and 5) were published in English language.

We excluded studies assessing physical inactivity, as physical inactivity cannot be equated with sedentary behaviour [7]. Furthermore, studies that considered ovarian cancer mortality or survival instead of ovarian cancer risk as the primary outcome were also unsuitable for analysis. We did not consider editorials, comments, letters, guidelines or news articles. All inclusion and exclusion criteria were defined prior to conducting the review.

\section{Search strategy}

We systematically searched the PubMed and Web of Science databases from inception to February 2020. Weekly updates provided by both databases were used and we reiterated our search on a monthly basis. Additionally, we manually reviewed the reference lists of suitable articles and consulted with experts in the field to obtain further relevant publications. Two authors (VB and CJ) developed the search term (Online Resource 2), which entailed sedentary behaviour and appropriate synonyms, as well as terms related to sedentary behaviours (e.g. leisure-time sitting, TV-viewing, computer use, transport time or reading), combined with keywords for ovarian neoplasms or site-specific cancer.

The first author (VB) screened titles and abstracts and retrieved full-text articles that met the inclusion criteria for further reading. The final decision about inclusion in the meta-analysis was made by two authors (VB and CJ) and disagreements were resolved by discussion with ML. If several reports regarding the same cohort study were found, we included the most recent publication.

\section{Data extraction}

The following data of each study was extracted by the first author (VB) and re-examined by the second author (CJ): first author's name, publication year, study design and name, size and age of study population, geographic region, followup time, number of incident cases, number of controls (if case-control design), histologic cancer type (total, epithelial, serous, non-serous), case ascertainment (self-report, medical record, linkage with state registries), sedentary behaviour domain (total, leisure-time, TV-viewing time, occupational time (hours per day spent sitting)), exposure ascertainment (self-administered questionnaire, standardised interview, job title assignment), unit of measurement (frequency, duration, intensity), and adjustment factors, risk estimates (RR, OR, HR) with corresponding 95\% CIs.

\section{Statistical methods}

Risk estimates were interpreted as relative risks $\left(R_{\mathrm{i}}\right)$. We computed the natural logarithms of relative risks $\left(\log \left(\mathrm{RR}_{\mathrm{i}}\right)\right)$ with their corresponding standard errors $\left(s_{i}=d_{i} / 1.96\right)$, where $d_{i}$ was defined as maximum of ( $\log$ (upper bound 95\% CI of $\left.\left.\mathrm{RR}_{\mathrm{i}}\right)-\log \left(\mathrm{RR}_{\mathrm{i}}\right)\right)$ and $\left(\log \left(\mathrm{RR}_{\mathrm{i}}\right)-(\log (\right.$ lower bound $95 \% \mathrm{CI}$ of $\mathrm{RR}_{\mathrm{i}}$ )). The logarithmic relative risks were weighted by $\omega_{\mathrm{i}}=1 /\left(\mathrm{s}_{\mathrm{i}}^{2}+\tau_{\mathrm{i}}^{2}\right)$ using a random-effects model, where $s_{i}$ describes the standard error of $\log \left(R R_{i}\right)$ and $\tau_{I}$ the restricted maximum likelihood estimate of the overall variance allowing for effect measure heterogeneity [12].

In the primary meta-analysis, we included one risk estimate per study. We preferred risk estimates primarily assessing total sitting time [13-15] or leisure-time sitting [16, 17] as exposure variables. We included two risk estimates of studies assessing solely TV-viewing time [18] or occupational sitting time [19], respectively. For all analyses, we chose the maximally adjusted risk estimate. We tested for heterogeneity using the Q- and $\mathrm{I}^{2}$-statistics [20].

We performed a priori defined stratified analyses with meta-regression random-effects meta-analysis and investigated the influence of study design (prospective cohort studies, case-control studies), geographic region (Asia, Europe, North America), sedentary behaviour domain (total, leisure-time, TV-viewing time, occupational time), exposure ascertainment (self-administered questionnaire, standardised interview, job title assignment) and adjustments for body mass index (BMI), family history of breast or ovarian cancer, parity, age at menarche, age at menopause, use of oral contraceptives, hormone therapy, education, alcohol use and smoking status. 
Publication bias was evaluated using a funnel plot [21], Begg's rank correlation test [22] and Egger's regression test [21]. Additionally, we performed outlier and influence diagnostics and leave-one-out analysis [23].

We calculated the E-Value to estimate how strong an unmeasured confounder would need to be to fully resolve a reported exposure-outcome association, above and beyond measured covariates [24]. As part of the calculation, we quantified the size of unobserved confounding able to nullify the mean risk ratio. As a meta-analytic extension of the E-Value, we calculated the unmeasured confounding strengths sufficient to allow $10 \%$ to $50 \%$ of studies with true RR above a meaningful scientific threshold (i.e., RR $>1.10$ ) to remain statistically significant [25].

All risk estimates were calculated with the corresponding 95\% CIs. P-values $<0.05$ were considered statistically significant. All statistical analyses were performed with the software R (version 3.5.1) [26], using the packages "robumeta" [27], "metafor" [12], "EValue" [25] and "MetaUtility" [28].

\section{Results}

\section{Study selection}

The systematic literature search identified 6074 publications, of which 6055 were found through electronic literature search (936 records through PubMed, 5119 records through Web of Science) and 19 through hand search or other sources (Fig. 1). After removal of duplicates, we screened titles and abstracts of 6062 publications. We assessed 23 full-text articles for eligibility. Of these, seven studies used incompatible study designs (meta-analyses or systematic reviews) [4, 8-10, 29-31], five studies investigated other exposure variables (physical activity or physical inactivity) [32-36], two studies did not provide sufficient data on ovarian cancer risk [37, 38] and two studies were updated by a more recent publication [39, 40] (Online Resource 3). After exclusion of these studies, a total of seven eligible articles, containing three prospective cohort studies $[14,16,18]$ and four case-control studies [13, 15, 17, 19], were included in our systematic review and meta-analysis.

\section{Study characteristics}

The main characteristics of the seven studies included are presented in Table 1. In total, the studies comprised 197,416 participants and 2060 ovarian cancer cases. Three studies provided risk estimates for total sitting time [13-15], three for leisure-time sitting [15-17] or TV-viewing time [14, 15, 18], respectively, and two studies for occupational sitting time [15, 19]. Two studies originated from Europe [17, 19], three from Asia $[13,15,18]$ and two from the United States
$[14,16]$. Three studies assessed sedentary behaviour through a self-administered questionnaire $[14,16,18]$, three studies used a standardised interview $[13,15,17]$, while one study used job titles [19]. The number of adjustment factors varied between two and 17 variables per study. All seven studies used daily duration of sedentary behaviour as exposure variable. The ovarian cancer cases were identified by self-report [16], medical records [13, 15-19], linkage to state cancer registries $[14,16,18]$ or death certificate [16]. We preferred risk ratios for total ovarian cancer $[16,18,19]$ or, if not provided, epithelial ovarian cancer [13-15, 17].

\section{Sedentary behaviour and ovarian cancer risk}

Our primary random-effects meta-analysis of seven risk estimates revealed a statistically significant positive association between high versus low level of sedentary behaviour and risk of ovarian cancer $(\mathrm{RR}=1.29,95 \% \mathrm{CI}=1.07-1.57)$ (Fig. 2). There was low heterogeneity among these studies $\left(\mathrm{I}^{2}=29.56 \%\right.$, P-heterogeneity $\left.=0.1523\right)$. The summary risk estimates were almost identical in cohort $(\mathrm{RR}=1.33$, 95\% $\mathrm{CI}=0.92-1.93)$ and case-control $(\mathrm{RR}=1.28,95 \%$ $\mathrm{CI}=0.98-1.68)$ studies, although the stratum-specific risk estimates were statistically non-significant and heterogeneity was modest in both cohort $\left(\mathrm{I}^{2}=41,4 \%\right.$, P-heterogeneity $=0.1316)$ and case-control $\left(\mathrm{I}^{2}=43,3 \%\right.$, P-heterogeneity $=0.0727$ ) studies.

\section{Stratified analyses and sensitivity analyses}

Stratified sub-analyses showed that the relation of sedentary behaviour to ovarian cancer risk was not modified by study design, geographic region, sedentary behaviour domain, exposure ascertainment, or various adjustment factors, including adjustments for BMI, family history of breast or ovarian cancer, parity, age at menarche, age at menopause, oral contraceptives, hormone therapy, education, alcohol use and smoking status (all P-difference >0.05) (Table 2).

Visual inspection of the funnel plot yielded no evidence for publication bias (Online Resource 4), as indicated by Egger's regression test $(\mathrm{P}=0.97)$ and Begg's correlation test $(\mathrm{P}=1.00)$.

Leave-one-out diagnostics and influence diagnostics of the seven included studies showed no relevant changes in summary risk estimates, spanning a range from $\mathrm{RR}=1.24$ $(95 \% \mathrm{CI}=1.02-1.51)$ to $\mathrm{RR}=1.38(95 \% \mathrm{CI}=1.12-1.70)$. For example, when the case-control study that used job title assignment and occupational sitting time as exposure variable [17] was omitted from the analysis, results showed a summary risk estimate of $\mathrm{RR}=1.32(95 \% \mathrm{CI}=1.08-1.60)$. When we excluded the prospective cohort study that assessed only TV-viewing time as exposure variable [18], the summary risk estimate was $\mathrm{RR}=1.27(95 \% \mathrm{CI}=1.04-1.55)$. 
6,055 Records identified through database

search

936 Records identified through PubMed 5,119 Records identified through Web of Science

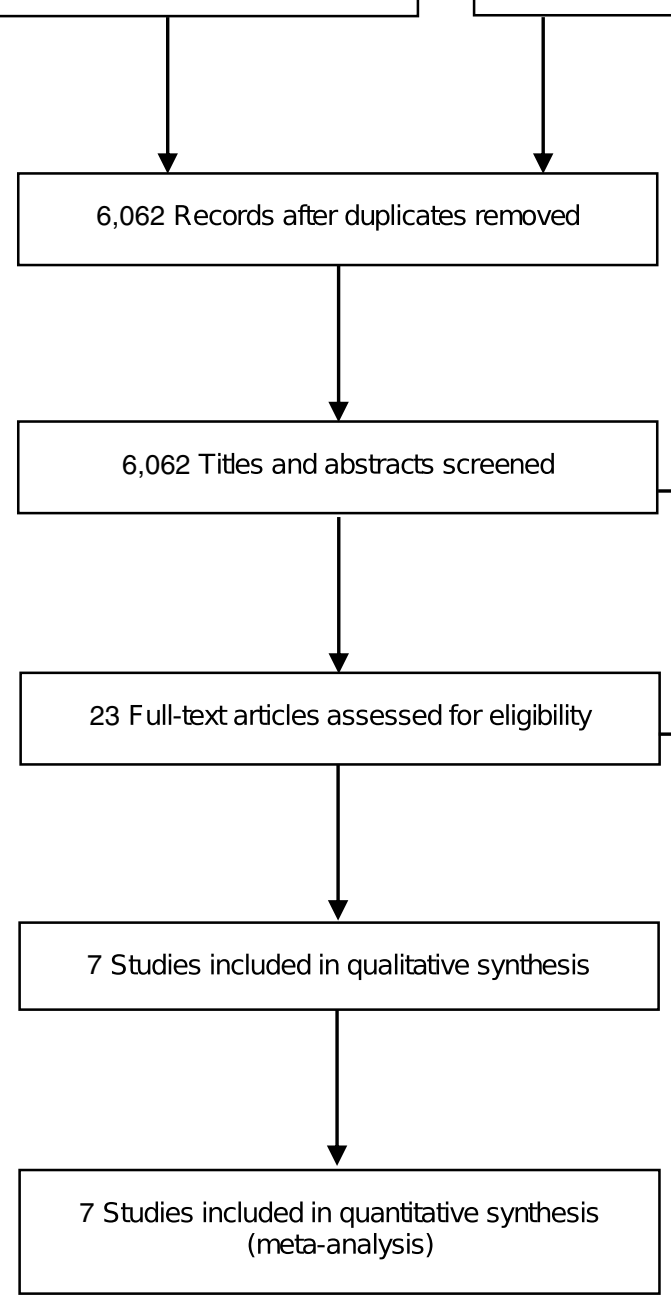

19 Additional records identified through other sources
6,039 Records excluded (not related to sedentary behaviour and ovarian cancer incidence)

16 Full-text articles excluded

7 Study designs were not suitable 5 Studies assessed other exposure variables

- $\quad$ Physical activity $(n=1)$

- $\quad$ Physical inactivity $(n=4)$

2 Studies had no data on ovarian cancer incidence

2 Studies were updated by a more recent study

Fig. 1 PRISMA flow diagram depicting the process of study selection for meta-analysis

The sensitivity analysis for unmeasured confounding showed that an unobserved confounder needed to be associated with both sedentary behaviour and ovarian cancer with a risk ratio of at least 1.90 to fully explain away the mean $R R$ of 1.29 , above and beyond the measured confounders, yet weaker confounding could not do so. To render the estimated risk ratio statistically non-significant, unobserved confounding strength associated with sedentary behaviour and ovarian cancer risk with a risk ratio of 1.34 would be necessary to move the lower confidence limit of 1.07 to include the null. Unobserved confounder strengths with RRs of 2.14, 1.96, $1.84,1.73$ and 1.63 in each meta-analysed study, respectively, would be necessary to reduce to less than $10 \%, 20 \%$, $30 \%, 40 \%$, and $50 \%$, respectively, the percentage of studies with true causal RRs above the meaningful scientific threshold of 1.10 (Online Resource 5).

\section{Discussion}

The results of our primary meta-analysis, including 2060 ovarian cancer cases from seven studies, showed a $29 \%$ increase in ovarian cancer risk with high versus low levels of sedentary behaviour. The association between sedentary behaviour and ovarian cancer risk was not modified by study design, geographic region, sedentary behaviour domain, exposure ascertainment, or adjustment for several confounders, including reproductive, hormonal, and lifestyle factors. 


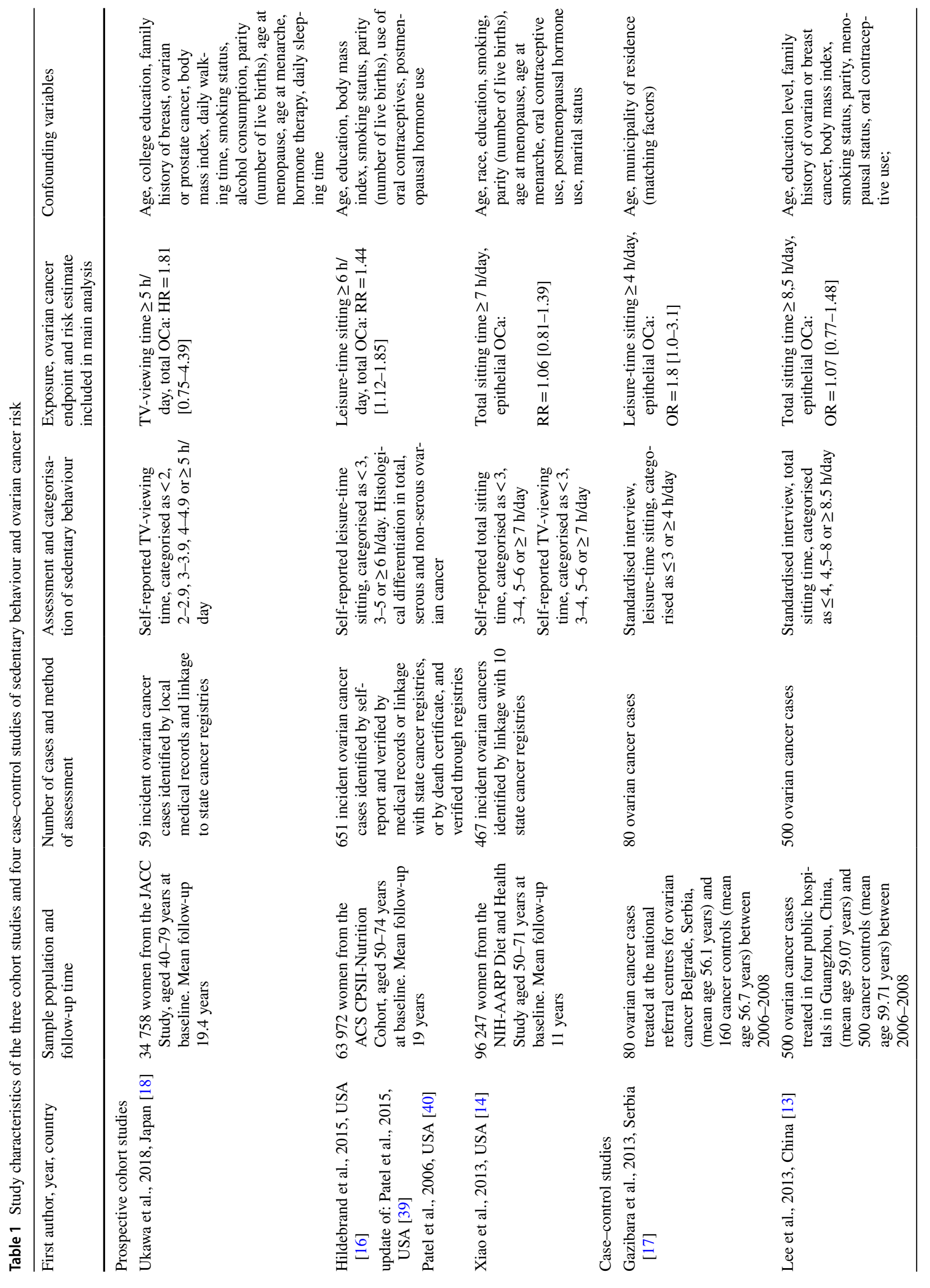




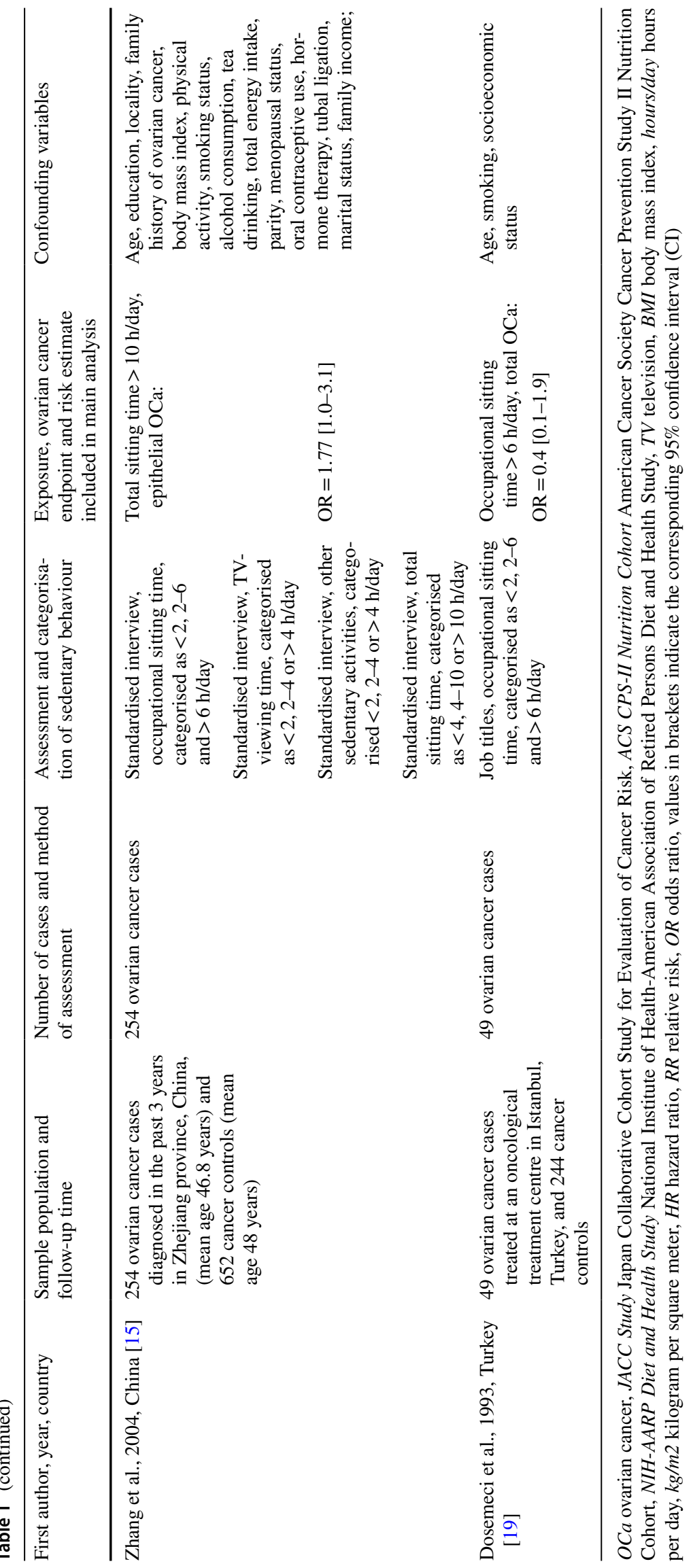




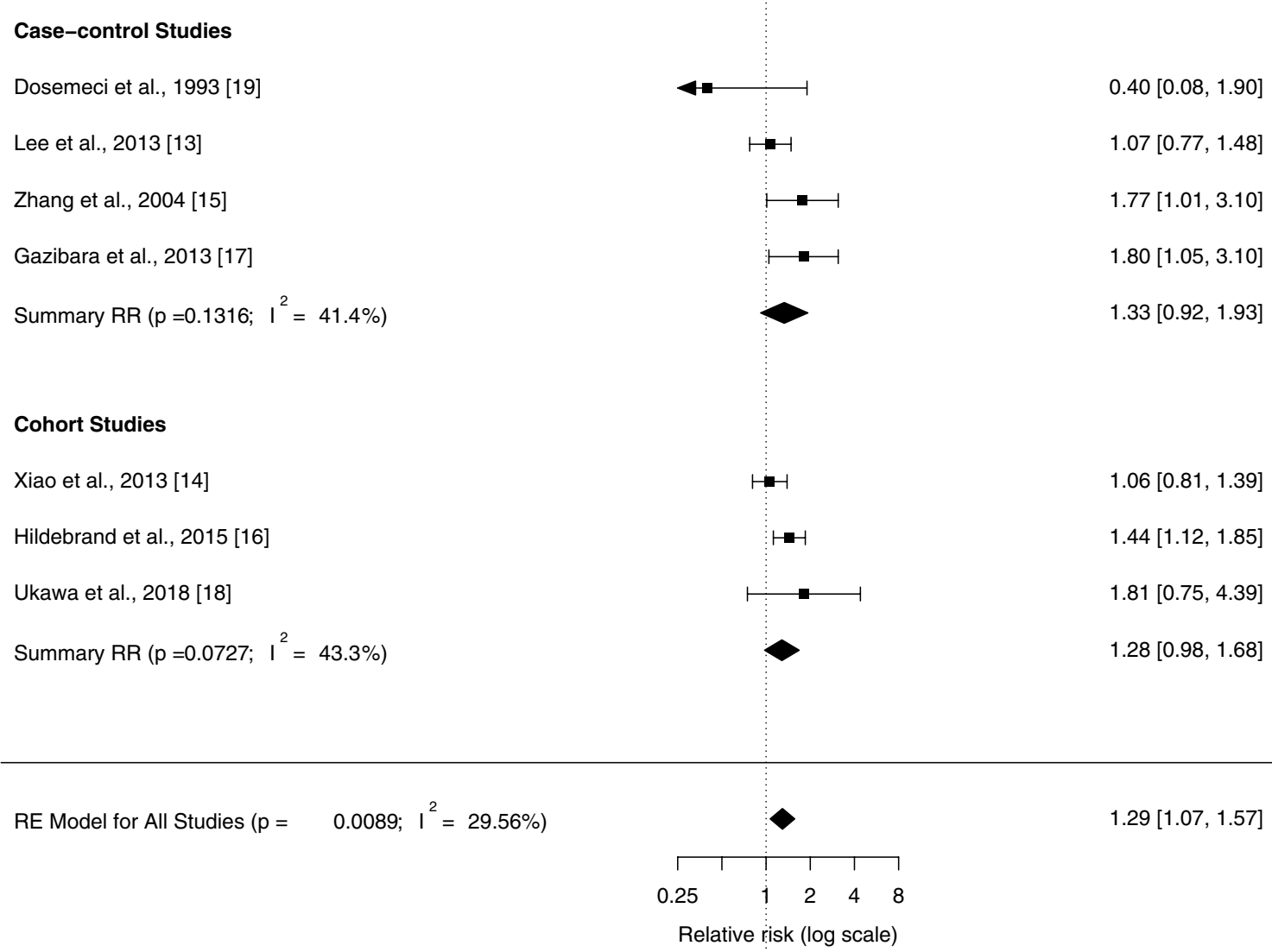

Fig. 2 Forest plot of random-effects meta-analysis of adjusted risk estimates of high versus low sedentary behaviour in relation to ovarian cancer risk. The black square and the respective line represent the risk estimate and corresponding $95 \%$ confidence interval (CI) for each study. The diamond represents the summary relative risk with

Our results update and expand the existing evidence on sedentary behaviour and ovarian cancer risk. Results from previous meta-analyses differed. Whereas some studies [4, 10] found positive yet statistically non-significant results, others $[8,9]$ reported a positive and statistically significant association. We saw the necessity to conduct a comprehensive analysis of all available studies, since the most recently published meta-analysis [8] contained several methodological shortcomings. First, several risk estimates included in that meta-analysis [8] could not be found in the original studies. Second, physical inactivity was used synonymously with sedentary behaviour as exposure variable. This led to the inclusion of one study that solely assessed physical inactivity [34]. We paid particular attention to not equate sedentary behaviour with absence of physical activity. Specifically, we defined physical inactivity as exclusion criterion and excluded four studies from our analysis (Online Resource the corresponding CI for ovarian cancer risk based on case-control studies, cohort studies, and all studies combined, respectively. P, $\mathrm{P}$-value (statistical significance); $\mathrm{I}^{2}$, heterogeneity among studies; RR, relative risk; RE Model, random effects mode

3). Third, three publications from the same cohort $[16,39$, 40] were included in the aforementioned meta-analysis [8], not only the most recent and updated one [16] but also the two prior publications [39, 40], which may have given that cohort undue weight, potentially distorting the results.

To our knowledge, the current study is the first metaanalysis on the association between sedentary behaviour and ovarian cancer risk that considered the E-Value as a parameter for unmeasured confounding, representing a novel methodological contribution to the existing evidence. The E-Value, a sensitivity analysis for unmeasured confounding, is a relatively new way to test the robustness of an association between exposure and outcome and to evaluate evidence for causation. Even though most of our included studies reduced confounding by adjusting the risk estimates for relevant factors, there is still concern about possible bias caused by uncontrolled confounding. Our results show that 
Table 2 Stratification criteria, relative risk, difference between included ovarian cancer risk studies and results of randomeffects meta-regression metaanalysis for each subgroup

\begin{tabular}{|c|c|c|c|c|c|}
\hline Stratification criteria & $\begin{array}{l}\text { Number of } \\
\text { included } \\
\text { RRs }\end{array}$ & $\begin{array}{l}\text { RR (high } \\
\text { versus low } \\
\text { SB) }\end{array}$ & $95 \% \mathrm{CI}$ & $\mathrm{I}^{2}(\%)$ & $\mathrm{P}($ diff $)$ \\
\hline Total incident ovarian cancer & 7 & 1.29 & $1.07,1.57$ & 29.56 & NA \\
\hline \multicolumn{6}{|l|}{ Study design } \\
\hline Prospective cohort studies & 3 & 1.28 & $0.98,1.68$ & 43.26 & \\
\hline Case-control studies & 4 & 1.33 & $0.92,1.93$ & 41.42 & 0.9021 \\
\hline \multicolumn{6}{|l|}{ Geographic region } \\
\hline Asia & 3 & 1.36 & $0.91,2.03$ & 39.80 & \\
\hline Europe & 2 & 1.02 & $0.24,4.26$ & 68.66 & \\
\hline North America & 2 & 1.24 & $0.92,1.68$ & 62.22 & 0.9104 \\
\hline \multicolumn{6}{|l|}{ Sedentary behaviour domain } \\
\hline Total & 3 & 1.13 & $0.93,1.38$ & 0.01 & \\
\hline Leisure-time & 3 & 1.34 & $0.95,1.90$ & 45.02 & \\
\hline TV-viewing time & 3 & 1.54 & $0.79,2.97$ & 51.78 & \\
\hline Occupational time & 2 & 1.06 & $0.23,4.83$ & 72.50 & 0.9018 \\
\hline \multicolumn{6}{|l|}{ Exposure ascertainment } \\
\hline Self-administered questionnaire & 3 & 1.28 & $0.98,1.68$ & 43.26 & \\
\hline Standardised interview & 3 & 1.42 & $0.97,2.07$ & 49.31 & \\
\hline Job title assignment & 1 & 0.40 & $0.08,1.90$ & NA & 0.3232 \\
\hline \multicolumn{6}{|l|}{ Adjustment for BMI } \\
\hline Adjusted for BMI & 4 & 1.36 & $1.08,1.71$ & 23.59 & \\
\hline Not adjusted for BMI & 3 & 1.18 & $0.71,1.95$ & 54.24 & 0.5031 \\
\hline \multicolumn{6}{|c|}{ Adjustment for family history of breast or ovarian cancer } \\
\hline Adjusted for family history & 3 & 1.36 & $0.91,2.03$ & 39.80 & \\
\hline Not adjusted for family history & 4 & 1.28 & $0.98,1.68$ & 43.24 & 0.8421 \\
\hline \multicolumn{6}{|l|}{ Adjustment for parity } \\
\hline Adjusted for parity & 5 & 1.27 & $1.04,1.54$ & 31.24 & \\
\hline Not adjusted for parity & 2 & 1.02 & $0.24,4.26$ & 68.66 & 0.6129 \\
\hline \multicolumn{6}{|l|}{ Adjustment for age at menarche / menopause } \\
\hline Adjusted for age at menarche / menopause & 2 & 1.16 & $0.78,1.74$ & 21.94 & \\
\hline Not adjusted for age at menarche / menopause & 5 & 1.36 & $1.09,1.70$ & 23.85 & 0.4008 \\
\hline \multicolumn{6}{|l|}{ Adjustment for oral contraceptives } \\
\hline Adjusted for oral contraceptives & 4 & 1.25 & $1.02,1.53$ & 37.31 & \\
\hline Not adjusted for oral contraceptives & 3 & 1.60 & $1.02,2.49$ & 0.00 & 0.3749 \\
\hline \multicolumn{6}{|l|}{ Adjustment for hormone therapy } \\
\hline Adjusted for hormone therapy & 4 & 1.35 & $1.05,1.73$ & 39.51 & \\
\hline Not adjusted for hormone therapy & 3 & 1.19 & $0.72,1.97$ & 51.36 & 0.6191 \\
\hline \multicolumn{6}{|l|}{ Adjustment for education } \\
\hline Adjusted for education & 5 & 1.27 & $1.04,1.54$ & 31.24 & \\
\hline Not adjusted for education & 2 & 1.02 & $0.24,4.26$ & 68.66 & 0.6129 \\
\hline \multicolumn{6}{|l|}{ Adjustment for alcohol use } \\
\hline Adjusted for alcohol use & 2 & 1.78 & $1.11,2.86$ & 0.00 & \\
\hline Not adjusted for alcohol use & 5 & 1.23 & $1.00,1.51$ & 33.90 & 0.1777 \\
\hline \multicolumn{6}{|l|}{ Adjustment for smoking status } \\
\hline Adjusted for smoking status & 6 & 1.24 & $1.02,1.51$ & 26.55 & \\
\hline Not adjusted for smoking status & 1 & 1.80 & $1.05,3.10$ & NA & 0.2468 \\
\hline
\end{tabular}

$R R$ relative risk, $S B$ sedentary behaviour, $C I$ confidence interval, $I^{2}$ heterogeneity among studies, $P($ diff $) \mathrm{P}$ value for difference in the result of moderator analysis, $N A$ not applicable, $B M I$ body mass index 
an unobserved confounder would have needed to be related to both the exposure and the outcome with a risk ratio of 1.90 to fully explain away the mean RR of 1.29 . Also, a confounder would have needed a risk ratio of 1.34 to render the risk estimate statistically non-significant. Although the observed E-value implies a true exposure-outcome association, we cannot fully rule out the existence of an unmeasured confounder associated with sedentary behaviour and ovarian cancer [24].

We were unable to examine whether prolonged sedentary behaviour affects ovarian cancer risk through an etiologic pathway involving obesity because none of the included studies provided risk estimates for different adiposity groups. However, several plausible hypotheses exist regarding obesity as a potential underlying biological mechanism. For example, time spent sedentary replaces time spent with physical activity, and high amounts of sedentary behaviour may coexist with increased energy supply [7], subsequently leading to weight gain and obesity [30, 41]. Current literature indicates that obesity not only increases the risk of ovarian cancer by itself, but may also act as an intermediate variable linking sedentary behaviour to ovarian cancer $[42,43]$. Sedentary behaviour and obesity are associated with a rise in sex hormone levels, particularly oestrogen and its metabolites produced by peripheral adipose tissue. This likely facilitates the development and progression of ovarian cancer through mitogenic and mutagenic effects [41, 44, 45]. Adipokines might also affect carcinogenesis through their roles in oestrogen biosynthesis and activity [44, 45]. In addition, sedentary behaviour and obesity-related insulin resistance, higher circulating levels of insulin and glucose, and enhancement of insulin-like growth factor 1 (IGF-1) are likely to boost cancer growth through involvement in cell differentiation, proliferation and apoptosis [41, 46]. Furthermore, systemic chronic inflammation is positively related to sedentary behaviour and obesity [47, 48]. Secretion of proinflammatory adipokines such as tumour necrosis factor- $\alpha$ (TNF- $\alpha$ ) and interleukin-6 (IL-6) by dysfunctional adipose tissue [49] and an increase in inflammatory factors, such as C-reactive protein (CRP) [50], are likely to promote cancer development [49]. Future studies of ovarian cancer risk stratified by levels of BMI are needed to formally test whether obesity or its metabolic sequelae represent intermediate steps in the causal pathway linking prolonged sedentary behaviour to ovarian cancer.

One challenge in interpreting the positive association between sedentary behaviour and ovarian cancer observed in our study is that there is currently limited evidence of an inverse relation of physical activity to ovarian cancer [51]. Numerous biologic mechanisms with cancer are believed to operate through an inverse association between physically active and sedentary behaviours. However, prolonged sedentary behaviour is distinct from the absence of physical activity, and time spent sedentary can co-exist with high levels of physical activity [7]. Also, sedentary behaviour may produce physiologic effects independent of those generated by moderate to vigorous activity. For example, several deleterious effects of prolonged sedentary time on cardiometabolic health have been reported for adults who met or exceeded the physical activity recommendations [52]. Thus, an increase in ovarian cancer risk associated with sedentary behaviour may in part be mediated by biologic mechanisms that do not involve an inverse relation of physical activity to ovarian cancer.

Our meta-analysis has numerous important strengths. We used unified and a priori defined criteria for our comprehensive literature search and extraction of relevant information from included studies. We conducted stratified analyses to identify potential sources of heterogeneity and provided precise and valid risk estimates throughout. Notably, we excluded studies that assessed physical inactivity instead of sedentary behaviour as the exposure variable. Importantly, sensitivity analysis for unmeasured cofounding, the E-Value has not yet been conducted in other meta-analyses covering the association between sedentary behaviour and ovarian cancer.

However, there are some limitations to our meta-analysis. Firstly, study designs were heterogenous (prospective cohort and case-control studies). As the number of existing prospective cohort studies on this topic is still small, more high-quality research is required to confirm our results. In addition, almost all studies used self-administered questionnaires $[14,16,18]$ or standardised interviews $[13,15,17]$ to assess sedentary behaviour instead of objective measures, such as accelerometry. This may have misclassified the true extent of sedentary behaviour [6, 53]. One study [19] used job title assignment to assess sedentary behaviour, which may have resulted in an inaccurate reflection of the actual time spent in sedentary pursuit, due to possible within-job variation, changes in job requirements over time or seasonal changes [54]. These limitations may have led to a certain level of measurement error, but risk estimates would have tended to be underestimated instead of overstated by nondifferential misclassification of sedentary behaviour levels [6]. Thus, the true detrimental effect of sedentary behaviour on ovarian cancer risk may be stronger than estimated in our analysis. The analysed data do not allow us to make any assumptions about the association between sedentary behaviour and tumour biology such as specific biological characteristics of ovarian cancer. We found some betweenstudy heterogeneity in the definition of high versus low levels of sedentary behaviour. Addressing this shortcoming by conducting dose-response analyses was not feasible due to the small number of included studies. Caution must be exercised when interpreting our results regarding potential effect measure modification of the sedentary behaviour and 
ovarian cancer relation because some of our stratified analyses were limited by small sample sizes. Also, insufficient data was the reason we were unable to assess the risk of each histological subtype separately, as only one included study analysed different subtypes of ovarian cancer [16]. Future research should take this into account and consider differentiating between histological subtypes.

In conclusion, this quantitative analysis of all available studies indicates that sedentary behaviour increases the risk of ovarian cancer. Our results represent an important step towards considering sedentary behaviour as a modifiable risk factor for ovarian cancer. Therefore, endeavours to reduce time spent sedentary throughout our everyday lives are to be encouraged on an individual and public health level.

Author contributions VB: Idea for the article, literature search, data analysis, drafting, critical revision and editing of the work. ML: Critical revision and supervision of the work. AS: Data analysis, critical revision of the work. FB: Critical revision of the work. CJ: Idea for the article, literature search, data analysis, critical revision and supervision of the work.

Funding Open Access funding enabled and organized by Projekt DEAL. This research did not receive any grant from funding organisations in the public, commercial, or not-for-profit sectors.

Data Availability Not applicable.

Code availability Available from the authors on request.

\section{Compliance with ethical standards}

Conflict of Interest The authors declare that they have no conflict of interest.

Open Access This article is licensed under a Creative Commons Attribution 4.0 International License, which permits use, sharing, adaptation, distribution and reproduction in any medium or format, as long as you give appropriate credit to the original author(s) and the source, provide a link to the Creative Commons licence, and indicate if changes were made. The images or other third party material in this article are included in the article's Creative Commons licence, unless indicated otherwise in a credit line to the material. If material is not included in the article's Creative Commons licence and your intended use is not permitted by statutory regulation or exceeds the permitted use, you will need to obtain permission directly from the copyright holder. To view a copy of this licence, visit http://creativecommons.org/licenses/by/4.0/.

\section{References}

1. Bray F, Ferlay J, Soerjomataram I, Siegel RL, Torre LA, Jemal A. Global cancer statistics 2018: GLOBOCAN estimates of incidence and mortality worldwide for 36 cancers in 185 countries. CA Cancer J Clin. 2018;68(6):394-424. https://doi.org/10.3322/ caac. 21492.

2. American Cancer Society. Cancer Facts \& Figures American Cancer Society, Atlanta. 2019.
3. Torre LA, Trabert B, DeSantis CE, Miller KD, Samimi G, Runowicz CD, et al. Ovarian cancer statistics, 2018. CA Cancer J Clin. 2018;68(4):284-96. https://doi.org/10.3322/caac.21456.

4. Schmid D, Leitzmann MF. Television viewing and time spent sedentary in relation to cancer risk: a meta-analysis. J Natl Cancer Inst. 2014;106(7):dju098. https://doi.org/10.1093/jnci/dju098.

5. Tremblay MS, Aubert S, Barnes JD, Saunders TJ, Carson V, Latimer-Cheung AE, et al. Sedentary Behavior Research Network (SBRN) - Terminology Consensus Project process and outcome. Int J Behav Nutr Phys Act. 2017;14(1):75. https://doi.org/10.1186/ s12966-017-0525-8.

6. Bauman AE, Petersen CB, Blond K, Rangul V, Hardy LL. The Descriptive Epidemiology of Sedentary Behaviour. In: Leitzmann MF, Jochem C, Schmid D, editors. Sedentary Behaviour Epidemiology. Cham: Springer Series on Epidemiology and Public Health; 2018. p. 73-106.

7. Owen N, Healy GN, Matthews CE, Dunstan DW. Too much sitting: the population health science of sedentary behavior. Exerc Sport Sci Rev. 2010;38(3):105-13. https://doi.org/10.1097/ JES.0b013e3181e373a2.

8. Lee J. Physical activity, sitting time, and the risk of ovarian cancer: A brief research report employing a meta-analysis of existing. Health Care Women Int. 2019;40(4):433-58. https:// doi.org/10.1080/07399332.2018.1505892.

9. Lynch BM, Mahmood S, Boyle T. Sedentary Behaviour and Cancer. In: Leitzmann MF, Jochem C, Schmid D, editors. Sedentary Behaviour Epidemiology. Cham: Springer Series on Epidemiology and Public Health; 2018. p. 245-98.

10. Shen D, Mao W, Liu T, Lin Q, Lu X, Wang Q, et al. Sedentary behavior and incident cancer: a meta-analysis of prospective studies. PLoS ONE. 2014;9(8):e105709. https://doi. org/10.1371/journal.pone.0105709.

11. Moher D, Liberati A, Tetzlaff J, Altman DG. Preferred reporting items for systematic reviews and meta-analyses: the PRISMA statement. J Clin Epidemiol. 2009;62(10):1006-12. https://doi. org/10.1016/j.jclinepi.2009.06.005.

12. Viechtbauer W. Conducting Meta-Analyses in $\mathrm{R}$ with the metafor Package 2010; 36(3): 48https://doi.org/10.18637/jss.v036. i03

13. Lee AH, Su D, Pasalich M, Wong YL, Binns CW. Habitual physical activity reduces risk of ovarian cancer: a case-control study in southern China. Prev Med. 2013;57(Suppl):S31-3. https://doi. org/10.1016/j.ypmed.2012.11.009.

14. Xiao Q, Yang HP, Wentzensen N, Hollenbeck A, Matthews CE. Physical activity in different periods of life, sedentary behavior, and the risk of ovarian cancer in the NIH-AARP diet and health study. Cancer Epidemiol Biomark Prev. 2013;22(11):2000-8. https://doi.org/10.1158/1055-9965.EPI-13-0154.

15. Zhang M, Xie X, Lee AH, Binns CW. Sedentary behaviours and epithelial ovarian cancer risk. Cancer Causes Control. 2004;15(1):83-9. https://doi.org/10.1023/B:CACO.0000016633 $.47025 .2 \mathrm{a}$.

16. Hildebrand JS, Gapstur SM, Gaudet MM, Campbell PT, Patel AV. Moderate-to-vigorous physical activity and leisure-time sitting in relation to ovarian cancer risk in a large prospective US cohort. Cancer Causes Control. 2015;26(11):1691-7. https://doi. org/10.1007/s10552-015-0656-7.

17. Gazibara T, Filipovic A, Kesic V, Kisic-Tepavcevic D, Pekmezovic T. Risk factors for epithelial ovarian cancer in the female population of Belgrade, Serbia: A case control study. Vojnosanit Pregl. 2013;70(12):1097-102. https://doi.org/10.2298/VSP11 0629030G.

18. Ukawa S, Tamakoshi A, Mori M, Ikehara S, Shirakawa T, Yatsuya $\mathrm{H}$, et al. Association between average daily television viewing time and the incidence of ovarian cancer: findings from 
the Japan Collaborative Cohort Study. Cancer Causes Control. 2018;29(2):213-9. https://doi.org/10.1007/s10552-018-1001-8.

19. Dosemeci M, Hayes RB, Vetter R, Hoover RN, Tucker M, Engin $\mathrm{K}$, et al. Occupational physical activity, socioeconomic status, and risks of 15 cancer sites in Turkey. Cancer Causes Control. 1993;4(4):313-21.

20. Higgins JP, Thompson SG. Quantifying heterogeneity in a metaanalysis. Stat Med. 2002;21(11):1539-58. https://doi.org/10.1002/ sim.1186.

21. Egger M, Davey Smith G, Schneider M, Minder C. Bias in meta-analysis detected by a simple, graphical test. BMJ. 1997;315(7109):629-34. https://doi.org/10.1136/ bmj.315.7109.629.

22. Begg CB, Mazumdar M. Operating characteristics of a rank correlation test for publication bias. Biometrics. 1994;50(4):1088-101.

23. Viechtbauer W, Cheung MW. Outlier and influence diagnostics for meta-analysis. Res Synth Methods. 2010;1(2):112-25. https ://doi.org/10.1002/jrsm.11.

24. VanderWeele TJ, Ding P. Sensitivity Analysis in Observational Research: Introducing the E-Value. Ann Intern Med. 2017;167(4):268. https://doi.org/10.7326/m16-2607.

25. Mathur MB, VanderWeele TJ. Sensitivity Analysis for Unmeasured Confounding in Meta-Analyses. J Am Stat Assoc. 2019;115:1-20. https://doi.org/10.1080/01621459.2018.1529598.

26. R Core Team. R: A language and environment for statistical computing. Vienna, Austria: R Foundation for Statistical Computing; 2018.

27. Fisher Z, Tipton E. robumeta: An R-package for robust variance estimation in meta-analysis. 2015.

28. Mathur MB, VanderWeele TJ. MetaUtility: Utility Functions for Conducting and Reporting Meta-Analysis. R package version 1.2.0. ed2019.

29. Friedenreich CM, Neilson HK, Lynch BM. State of the epidemiological evidence on physical activity and cancer prevention. Eur J Cancer. 2010;46(14):2593-604. https://doi.org/10.1016/j. ejca.2010.07.028.

30. Lynch BM. Sedentary behavior and cancer: a systematic review of the literature and proposed biological mechanisms. Cancer Epidemiol Biomark Prev. 2010;19(11):2691-709. https://doi. org/10.1158/1055-9965.EPI-10-0815.

31. Permuth-Wey J, Sellers TA. Epidemiology of ovarian cancer. Methods Mol Biol. 2009;472:413-37. https://doi. org/10.1007/978-1-60327-492-0_20.

32. Abbott SE, Bandera EV, Qin B, Peres LC, Moorman PG, Barnholtz-Sloan J, et al. Recreational physical activity and ovarian cancer risk in African American women. Cancer Med. 2016;5(6):1319-27. https://doi.org/10.1002/cam4.677.

33. Cannioto R, LaMonte MJ, Risch HA, Hong C-C, SuchestonCampbell LE, Eng KH, et al. Chronic recreational physical inactivity and epithelial ovarian cancer risk: evidence from the ovarian cancer association consortium. Cancer Epidemiol Biomark Prev. 2016;25(7):1114-24. https://doi.org/10.1158/1055-9965. EPI-15-1330.

34. Cannioto RA, Lamonte MJ, Kelemen LE, Risch HA, Eng KH, Minlikeeva AN, et al. Recreational physical inactivity and mortality in women with invasive epithelial ovarian cancer: evidence from the Ovarian Cancer Association Consortium. Br J Cancer. 2016;115(1):95-101. https://doi.org/10.1038/bjc.2016.153.

35. Minlikeeva AN, Cannioto R, Jensen A, Kjaer SK, Jordan SJ, Diergaarde B, et al. Joint exposure to smoking, excessive weight, and physical inactivity and survival of ovarian cancer patients, evidence from the Ovarian Cancer Association Consortium. Cancer causes \& control : CCC. 2019;30(5):537-47. https://doi. org/10.1007/s10552-019-01157-3.
36. Park S, Kim Y, Shin H-R, Lee B, Shin A, Jung K-W, et al. Population-attributable causes of cancer in Korea: obesity and physical inactivity. PLoS ONE. 2014;9(4):e90871-e. https://doi. org/10.1371/journal.pone.0090871.

37. Biswas A, Oh PI, Faulkner GE, Bajaj RR, Silver MA, Mitchell MS, et al. Sedentary Time and Its Association With Risk for Disease Incidence, Mortality, and Hospitalization in Adults. Ann Intern Med. 2015;162(2):123. https://doi.org/10.7326/m14-1651.

38. Rangul V, Sund ER, Mork PJ, Røe OD, Bauman A. The associations of sitting time and physical activity on total and sitespecific cancer incidence: Results from the HUNT study, Norway. PLoS ONE. 2018;13(10):e0206015. https://doi.org/10.1371/journ al.pone.0206015.

39. Patel AV, Hildebrand JS, Campbell PT, Teras LR, Craft LL, McCullough ML, et al. Leisure-Time Spent Sitting and Site-Specific Cancer Incidence in a Large U.S. Cohort. Cancer Epidemiol Biomark Prev. 2015;24(9):1350-9. https://doi.org/10.1158/10559965.EPI-15-0237.

40. Patel AV, Rodriguez C, Pavluck AL, Thun MJ, Calle EE. Recreational physical activity and sedentary behavior in relation to ovarian cancer risk in a large cohort of US women. Am J Epidemiol. 2006;163(8):709-16. https://doi.org/10.1093/aje/kwj098.

41. Jochem C, Wallmann-Sperlich B, Leitzmann MF. The influence of sedentary behavior on cancer risk: epidemiologic evidence and potential molecular mechanisms. Current Nutrition Reports. 2019;8(3):167-74. https://doi.org/10.1007/s13668-019-0263-4.

42. Collaborative Group on Epidemiological Studies of Ovarian C. Ovarian cancer and body size: individual participant metaanalysis including 25,157 women with ovarian cancer from 47 epidemiological studies. PLoS medicine. 2012;9(4):e1001200-e. doi:https://doi.org/10.1371/journal.pmed.1001200.

43. World Cancer Research Fund/American Institute for Cancer Research. Continuous Update Project Expert Report 2018. Diet, nutrition, physical activity and ovarian cancer 2018.

44. Dallal CM, Brinton LA, Matthews CE, Pfeiffer RM, Hartman TJ, Lissowska J et al. Association of Active and Sedentary Behaviors with Postmenopausal Estrogen Metabolism. 2016;48(3):439-48. https://doi.org/10.1249/mss.0000000000000790

45. Schüler S, Ponnath M, Engel J, Ortmann O. Ovarian epithelial tumors and reproductive factors: a systematic review. Arch Gynecol Obstet. 2013;287(6):1187-204. https://doi.org/10.1007/ s00404-013-2784-1.

46. Kahn BB, Flier JS. Obesity and insulin resistance. Journal of Clinical Investigation. 2000;106(4):473-81. https://doi.org/10.1172/ jci10842.

47. Henson J, Yates T, Edwardson CL, Khunti K, Talbot D, Gray LJ, et al. Sedentary time and markers of chronic low-grade inflammation in a high risk population. PLoS ONE. 2013;8(10):e78350. https://doi.org/10.1371/journal.pone.0078350.

48. Renehan AG, Zwahlen M, Egger M. Adiposity and cancer risk: new mechanistic insights from epidemiology. Nat Rev Cancer. 2015;15(8):484-98. https://doi.org/10.1038/nrc3967.

49. Van Kruijsdijk RCM, Van Der Wall E, Visseren FLJ. Obesity and cancer: the role of dysfunctional adipose tissue. Cancer Epidemiol Biomark Prev. 2009;18(10):2569-78. https://doi. org/10.1158/1055-9965.epi-09-0372.

50. Fogarty AW, Glancy C, Jones S, Lewis SA, McKeever TM, Britton JR. A prospective study of weight change and systemic inflammation over 9 y. Am J Clinical Nutrition. 2008;87(1):30-5. https ://doi.org/10.1093/ajcn/87.1.30.

51. Physical Activity Guidelines Advisory Committee. Physical Activity Guidelines Advisory Committee Scientific Report. Washington, DC: U.S. Department of Health and Human Services. 2018. 
52. Tremblay MS, Colley RC, Saunders TJ, Healy GN, Owen N. Physiological and health implications of a sedentary lifestyle. Appl Physiol Nutr Metab. 2010;35(6):725-40. https://doi.org/10.1139/ h10-079.

53. Cerin E, Cain KL, Oyeyemi AL, Owen N, Conway TL, Cochrane T, et al. Correlates of Agreement between Accelerometry and Self-reported Physical Activity. Med Sci Sports Exerc. 2016;48(6):1075-84. https://doi.org/10.1249/MSS.0000000000 000870 .
54. LaPorte RE, Montoye HJ, Caspersen CJ. Assessment of physical activity in epidemiologic research: problems and prospects. Public Health Rep. 1985;100(2):131-46.

Publisher's Note Springer Nature remains neutral with regard to jurisdictional claims in published maps and institutional affiliations. 